\title{
A Decomposition Algorithm for Solving a Three Level Large Scale linear Programming Problem
}

\author{
T. I. Sultan ${ }^{1}$, O. E. Emam ${ }^{1, *}$ and A. A. Abohany ${ }^{2}$ \\ ${ }^{1}$ Department of Information Systems, Faculty of Computer Science and Information, Helwan University, P.O. Box 11795, Egypt \\ ${ }^{2}$ Director of Electronic Document Management Systems, Human Resources Behalf, Telecom Egypt, Smart Village, B7, Egypt
}

Received: 18 Aug. 2013, Revised: 15 Nov. 2013, Accepted: 16 Nov. 2013

Published online: 1 Sep. 2014

\begin{abstract}
This paper presents a three level large scale linear programming problem in which the objective functions at every level are to be maximized. A three level programming problem can be thought as a static version of the Stackelberg strategy. An algorithm for solving a three planner model and a solution method for treating this problem are suggested. At each level we attempt to optimize its problem separately as a large scale programming problem using Dantzig and Wolfe decomposition method. Therefore, we handle the optimization process through a series of sub problems that can be solved independently. Finally, a numerical example is given to clarify the main results developed in this paper.
\end{abstract}

Keywords: Large scale problems; linear programming; three-level programming; decomposition algorithm. MSC 2000: 90C06; 90C05; 90C99.

\section{Introduction}

Multilevel optimization problems have attracted considerable attention from the scientific and economic community in recent years. The multilevel system has extensive existences in management fields. Usually, this kind of problems can be solved by using different mathematical programming techniques ([8], [11]).

Most studies in multilevel field are focused on bi-level problem $([10,13,14,15,16,17])$. In [10], Emam proposed an algorithm for solving bi-level integer multi-objective fractional programming problem. At the first phase of the solution algorithm, it begin by finding the convex hull of its original set of constraints then simplifying the equivalent problem by transforming it into a separate multi-objective decision-making problem and finally solving the resulted problem by using the $\varepsilon$-constraint method.

Pramanik and Banerjee presented an approach to deal with fuzzy goal programming approach to solve chance constrained quadratic bi-level programming problem [13]. The presented approach convert the chance constraints into equivalent deterministic constraints with prescribed distribution functions and confidence levels. Then a quadratic membership function by using individual best solution based on first order Taylor's series is formed.

In large scale programming which closely describes and represents the real world decision situations, various factors of the real system should be reflected in the description of the objective function and constraints. Naturally these objective function and constraints involve many parameters and the experts may assign them different values $([1,2,3,4])$.

After the publication of the Dantzig and Wolfe decomposition method [7], there have been numerous subsequent works on large scale linear and nonlinear programming problems with block angular structure ([1, $6,12])$.

Abo Sinna et al. [4] extended the technique for order preference by similarity ideal solution (TOPSIS) to solve multi-objective large scale non-linear programming problem. Compromise (TOPSIS) control minimizes the measure of distance. The concept of a membership function of fuzzy set theory was used to represent the satisfaction level for both criteria. El-Sawy et al. [9] introduced an algorithm for decomposing the parametric space in large scale linear vector optimization problems under fuzzy environment.

Recently, notable studies have been done in the area of multi-level and multi-objective large scale programming

\footnotetext{
*Corresponding author e-mail: emam_o_e@yahoo.com
} 
problems $([3,4,6,12])$.

Benzi et al. [6] developed and compared multi-level algorithms for solving large scale bound constrained nonlinear problems via interior point methods. It show how a multilevel continuation strategy can be used to obtain good initial guesses for each nonlinear iteration. A minimal surface problem is used to illustrate the various approaches.

Osman et al. [12] presented a method for solving a special class of large scale fuzzy multi-objective integer problems depending on the decomposition algorithm. Furthermore Abo-Sinna and Abou-Elenin extended TOPSIS for solving large scale multiple objective programming involving fuzzy parameters [3]. These fuzzy parameters are characterized as fuzzy numbers, the $\alpha$-Pareto optimality is introduced by extending the ordinary Pareto optimality on the basis of the $\alpha$-level sets of fuzzy numbers.

This paper is organized as follows: we start in Section 2 by formulating the model of a three level large scale linear programming problem. In Section 3, the decomposition method of large scale three level linear programming problem is presented. An algorithm followed by a flowchart for solving a three level linear programming is suggested in Section 4 and Section 5. In addition, a numerical example is provided in Section 6 to clarify the results and the solution algorithm. Finally, conclusion and future works are reported in Section 7.

\section{Problem Formulation and Solution Concept}

The three level large scale linear programming problem (TLLSLPP) may be formulated as follows:

[First Level]

$$
\underset{x_{1}, x_{2}}{\operatorname{Max}} F_{1}(x)=\operatorname{Max}_{x_{1}, x_{2}} c_{1 j} x_{j},
$$

[Second Level]

Where $x_{3}, \ldots, x_{m}$ solves

$$
\underset{x_{3}, x_{4}}{\operatorname{Max}} F_{2}(x)=\operatorname{Max}_{x_{3}, x_{4}} c_{2 j} x_{j},
$$

[Third Level]

$$
\text { Where } x_{5}, \ldots, x_{m} \text { solves }
$$

$$
\underset{x_{5}, x_{6}}{\operatorname{Max}_{3}} F_{3}(x)=\operatorname{Max}_{x_{5}, x_{6}} c_{3 j} x_{j},
$$

Subject to

$$
\text { Where } x_{7}, \ldots, x_{m} \text { solves }
$$

$$
x \in G \text {. }
$$

Where

$$
\begin{aligned}
G=\left\{a_{01} x_{1}+a_{02} x_{2}\right. & +a_{0 m} x_{m} \\
d_{1} x_{1} & \leq b_{0} \\
& \leq b_{1} \\
d_{2} x_{2} & \leq b_{2} \\
& \\
& d_{m} x_{m} \leq b_{m}, \\
x_{1}, \ldots, x_{m} & \geq 0\}
\end{aligned}
$$

In the above problem (1)-(4), $x_{j} \in R,(j=1,2, \ldots, m)$ be a real vector variables, $G$ is the large scale linear constraint set where, $b=\left(b_{0}, \ldots, b_{m}\right)^{T}$ is $(m+1)$ vector, and $a_{01}, \ldots, a_{0 m}, d_{1}, d_{m}$ are constants. Therefore $F_{i}: R^{m} \rightarrow R,(i=1,2,3)$ be the first level objective function, the second level objective function, and the third level objective function, respectively. Moreover, the first level decision maker (FLDM) has $x_{1}, x_{2}$ indicating the first decision level choice, the second level decision maker (SLDM) and the third level decision maker (TLDM) have $x_{3}, x_{4}$ and $x_{5}, x_{6}$ indicating the second decision level choice and the third decision level choice, respectively.

Definition 1. For any $\left(x_{1}, x_{2} \in G_{1}=\left\{x_{1}, x_{2} \mid\right.\right.$ $\left.\left.\left(x_{1}, \ldots, x_{m}\right) \in G\right\}\right)$ given by FLDM and $\left(x_{3}, x_{4} \in G_{2}=\left\{x_{3}, x_{4} \mid\left(x_{1}, \ldots, x_{m}\right) \in G\right\}\right)$ given by SLDM, if the decision-making variable $\left(x_{5}, x_{6} \in G_{3}=\right.$ $\left.\left\{x_{5}, x_{6} \mid\left(x_{1}, \ldots, x_{m}\right) \in G\right\}\right)$ is the Pareto optimal solution of the TLDM, then $\left(x_{1}, \ldots, x_{m}\right)$ is a feasible solution of TLLSLPP.

Definition 2. If $x^{*} \in R^{m}$ is a feasible solution of the TLLSLPP; no other feasible solution $x \in G$ exists, such that $F_{1}\left(x^{*}\right) \leq F_{1}(x)$; so $x^{*}$ is the Pareto optimal solution of the TLLSLPP.

\section{Decomposition algorithm for the three level large scale linear programming problem}

The three level large scale linear programming problem is solved by adopting the leader-follower Stakelberg strategy combine with Dantzig and Wolf decomposition method $([3,7,9])$. One first gets the optimal solution that is acceptable to FLDM using the decomposition method to break the large scale problem into n-sub problems that can be solved directly.

The decomposition principle is based on representing the TLLSLPP in terms of the extreme points of the sets $d_{j} x_{j} \leq b_{j}, x_{j} \geq 0, j=1,2, \ldots, m$. To do so, the solution space described by each $d_{j} x_{j} \leq b_{j}, x_{j} \geq 0, j=1,2, \ldots, m$ must be bounded and closed.

Then by inserting the FLDM decision variable to the SLDM for him/her to seek the optimal solution using Dantzig and Wolf decomposition method [7], then the decomposition method break the large scale problem into n-sub problems that can be solved directly.

Finally the TLDM do the same action till he obtains the optimal solution of his problem which is the optimal solution to TLLSLPP.

Theorem 1. The decomposition algorithm terminates in a finite number of iterations, yielding a solution of the large scale problem.

To prove theorem 1 above, the reader is referred to [7]. 


\subsection{The First-Level Decision-Maker (FLDM) Problem}

The first-level decision-maker problem of the TLLSLPP is as follows:

[First Level]

$$
\operatorname{Max}_{1}(x)=\operatorname{Max} \sum_{j=1}^{m} c_{1 j} x_{j}
$$

Subject to

$$
x \in G \text {. }
$$

To obtain the optimal solution of the FLDM problem; suppose that the extreme points of $d_{j} x_{j} \leq b_{j}, x_{j} \geq 0$ are defined as $\hat{x}_{j k}, k=1,2,3$, where $x_{j}$ defined by:

$$
x_{j}=\sum_{k=1}^{k_{j}} \beta_{j k} \hat{x}_{j k}, j=1, \ldots, m .
$$

$$
\text { and } \beta_{j k} \geq 0 \text {, for all } k \text { and } \sum_{k=1}^{k_{j}} \beta_{j k}=1 \text {. }
$$

Now, the FLDM problem in terms of the extreme points to obtain the following master problem of the FLDM are formulated as stated in [7]:

$$
\operatorname{Max} \sum_{k=1}^{k_{1}} c_{11} \hat{x}_{1 k} \beta_{1 k}+\sum_{k=1}^{k_{2}} c_{12} \hat{x}_{2 k} \beta_{2 k}+\cdots+\sum_{k=1}^{k_{n}} c_{1 n} \hat{x}_{n k} \beta_{n k}
$$

Subject to

$$
\begin{aligned}
& \sum_{k=1}^{k_{1}} a_{01} \hat{x}_{1 k} \beta_{1 k}+\sum_{k=1}^{k_{2}} a_{02} \hat{x}_{2 k} \beta_{2 k}+\cdots+\sum_{k=2}^{k_{n}} a_{0 n} \hat{x}_{n k} \beta_{n k} \leq b_{0}, \\
& \sum_{k=1}^{k_{1}} \beta_{1 k}=1 \\
& \sum_{k=1}^{k_{2}} \beta_{2 k}=1 \text {, } \\
& \sum_{k=1}^{k_{n}} \beta_{n k}=1 \text {, } \\
& \beta_{j k} \geq 0 \text {, for all } j \text { and } k \text {. }
\end{aligned}
$$

The new variables in the FLDM problem are $\beta_{j k}$ which determined using Balinski's algorithm [5]. Once their optimal values $\beta_{j k}^{*}$ are obtained, then the optimal solution to the original problem can be found by back substitution as follow:

$$
x_{j}=\sum_{k=1}^{k_{1}} \beta_{j k}^{*} \hat{x}_{j k}, j=1,2,3 .
$$

It may appear that the solution of the FLDM problem requires prior determination of all extreme points $\hat{x}_{j k}$. To solve the FLDM problem by the revised simplex method, it must determine the entering and leaving variables at each iteration. Let us start first with the entering variables.
Given $C_{B}$ and $B^{-1}$ of the current basis of the FLDM problem, then for non-basic $\beta_{j k}$ :

$$
z_{j k}-c_{j k}=C_{B} B^{-1} P_{j k}-C_{j k}
$$

Where

$$
C_{j k}=C_{j} \hat{x}_{j k} \text { and } P_{j k}=\left[\begin{array}{c}
a_{j} \hat{x}_{j k} \\
0 \\
1 \\
0
\end{array}\right]
$$

Now, to decide which of the variables $\beta_{j k}$ should enter the solution it must determine:

$$
z_{j k}^{*}-c_{j k}^{*}=\min \left\{z_{j k}-c_{j k}\right\}
$$

Consequently, if $z_{j k}^{*}-c_{j k}^{*} \leq 0$, then according to the maximization optimality condition, $\beta_{j k}^{*}$ must enter the solution; otherwise, the optimal has been reached.

\subsection{The Second-Level Decision-Maker (SLDM) Problem}

Secondly, according to the mechanism of the TLLSLPP, the FLDM variables $x_{1}^{F}, x_{2}^{F}$ should be given to the SLDM; hence, the SLDM problem can be written as follows:

$$
\operatorname{Max}_{2}(x)=\operatorname{Max} \sum_{j=1}^{m} c_{2 j} x_{j}
$$

Subject to

$$
\left(x_{1}^{F}, x_{2}^{F}, \ldots, x_{m}\right) \in G
$$

To obtain the optimal solution of the SLDM problem; the SLDM solves his master problem by the decomposition method [7] as the FLDM.

\subsection{The Third-Level Decision-Maker (TLDM) Problem}

Finally, according to the mechanism of the TLLSLPP, the SLDM variables $x_{1}^{F}, x_{2}^{F}, x_{3}^{S}, x_{4}^{S}$ should be given to the TLDM; hence, the TLDM problem can be written as follows:

$$
\operatorname{Max}_{3}(x)=\operatorname{Max} \sum_{j=1}^{m} c_{3 j} x_{j}
$$

Subject to

$$
\left(x_{1}^{F}, x_{2}^{F}, x_{3}^{S}, x_{4}^{S}, \ldots, x_{m}\right) \in G .
$$

To obtain the optimal solution of the TLDM problem; the TLDM solves his master problem by the decomposition method [13] as the FLDM and SLDM.

Now the optimal solution $\left(x_{1}^{F}, x_{2}^{F}, x_{3}^{S}, x_{4}^{S}, x_{5}^{T}, x_{6}^{T}, \ldots, x_{m}^{T}\right)$ of the TLDM is the optimal solution of the TLLSLPP. 


\section{An algorithm for solving TLLSLPP}

A solution algorithm to solve three-level large scale linear programming problem (TLLSLPP) is described in a series of steps. This algorithm overcome the complexity nature of the three level large scale linear programming problem, and uses the constraint method of the three level optimization to facility the large scale linear constraints nature. Inserting the variables value of every higher level decision maker to his lower level decision maker break the difficulty faces the TLLSLPP.

The suggested algorithm can be summarized in the following manner:

\section{Step 1.}

Start with the FLDM problem and go to Step 2.

\section{Step 2.}

Convert the master problem in terms of extreme points of the sets $d_{j} x_{j} \leq b_{j}, x_{j} \geq 0, j=1,2,3$.

\section{Step 3.}

Determine the extreme points $x_{j}=\sum_{k=1}^{k_{j}} \beta_{j k} \hat{x}_{j k}$, $j=1,2,3$ using Balinski’s algorithm [5].

\section{Step 4.}

Set $k=1$.

\section{Step 5.}

Compute $z_{j k}-c_{j k}=C_{B} B^{-1} P_{j k}-C_{j k}$, go to Step 6 .

\section{Step 6.}

If $z_{j k}^{*}-c_{j k}^{*} \leq 0$, then go to Step 7; otherwise, the optimal solution has been reached, go to Step 8 .

\section{Step 7.}

Set $k=k+1$, go to Step 4 .

\section{Step 8.}

If the SLDM obtain the optimal solution go to Step 11 , otherwise go to Step 9.

\section{Step 9.}

Set $\left(x_{1}, x_{2}\right)=\left(x_{1}^{F}, x_{2}^{F}\right)$ to the SLDM constraints, go to Step 10.

\section{Step 10.}

The SLDM formulate his problem, go to Step 2.

\section{Step 11.}

If the TLDM obtain the optimal solution go to Step 14 , otherwise go to Step 12.

\section{Step 12.}

Set $\left(x_{1}, x_{2}, x_{3}, x_{4}\right)=\left(x_{1}^{F}, x_{2}^{F}, x_{3}^{S}, x_{4}^{S}\right)$ to the TLDM constraints, go to Step 13 .

\section{Step 13.}

The TLDM formulate his problem, go to Step 2.

\section{Step 14.}

$\left(x_{1}^{F}, x_{2}^{F}, x_{3}^{S}, x_{4}^{S}, x_{5}^{T}, x_{6}^{T}, \ldots, x_{m}^{T}\right)$ is as an optimal solution for three-level large scale linear programming problem, then stop.

\section{A flowchart for solving TLLSLPP}

A flowchart to explain the suggested algorithm for solving a three-level large scale linear programming problem is described as shown in figure 1 .

\section{Numerical example}

To demonstrate the solution for (TLLSLPP), let us consider the following problem:

[First Level]

$\operatorname{Max}_{x_{1}, x_{2}} F_{1}(x)=\underset{x_{1}, x_{2}}{\operatorname{Max}} 5 x_{1}+4 x_{2}+x_{5}+x_{6}$,
Where $x_{3}, x_{4}, x_{5}, x_{6}$ solves

[Second Level]

$\operatorname{Max}_{x_{3}, x_{4}} F_{2}(x)=\operatorname{Max}_{x_{3}, x_{4}} 8 x_{3}+4 x_{4}+x_{5}+x_{6}$,

Where $x_{5}, x_{6}$ solves

[Third Level]

$\operatorname{Max}_{x_{5}, x_{6}} F_{3}(x)=\operatorname{Max}_{x_{5}, x_{6}} \underset{x}{ } x_{5}+6 x_{6}$,

Subject to

$$
\begin{aligned}
& x_{1}+x_{2}+x_{3}+x_{4}+x_{5}+x_{6} \leq 50, \\
& 2 x_{1}+x_{2} \leq 40, \\
& 5 x_{3}+x_{4} \leq 12, \\
& x_{5}+x_{6} \leq 20, \\
& x_{5}+5 x_{6} \leq 80, \\
& x_{1}, x_{2}, x_{3}, x_{4}, x_{5}, x_{6} \geq 10 .
\end{aligned}
$$

Firstly, the FLDM problem formulation as follows : $\operatorname{Max}_{1}(x)=\operatorname{Max} 5 x_{1}+4 x_{2}+x_{5}+x_{6}$,

Subject to $x \in G$.

1. Convert the FLDM problem in terms of extreme points of the sets $d_{j} x_{j} \leq b_{j}, x_{j} \geq 0, j=1,2,3$.

2. Determine the extreme points $x_{j}=\sum_{k=1}^{k_{j}} \beta_{j k} \hat{x}_{j k}$, $j=1,2,3$ using Balinski's algorithm [5].

3 . Set $k=1$, so the slack variable $x_{7}$ convert common 


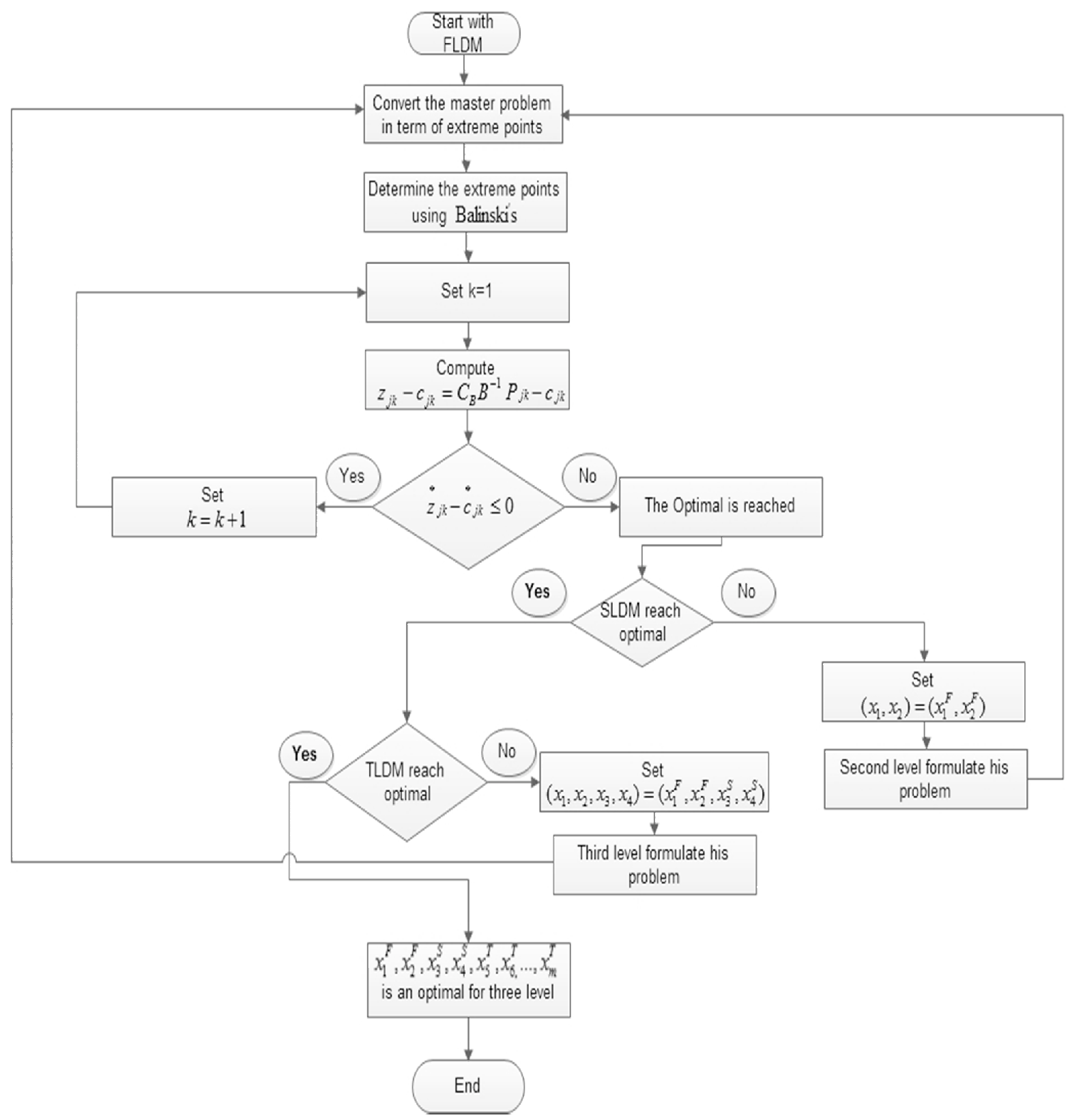

Fig. 1: Algorithm for solving a three-level large scale linear programming problem

constraint into equation $x_{8}, x_{9}, x_{10}$ are artificial variables as :

$$
x_{1}+x_{2}+x_{3}+x_{4}+x_{5}+x_{6}+x_{7}=50 \text {. }
$$

Let us identify Iteration 0 as:

$X_{B}=\left(x_{7}, x_{8}, x_{9}, x_{10}\right)^{T}, \quad X_{B}=(50,1,1,1)^{T}$, $C_{B}=(0,-M,-M,-M), B=1, B^{-1}=1$.

Now iteration 1 for sub problem 1 where $\mathrm{j}=1$ is :

$Z_{1}-C_{1}=C_{B} B^{-1}=\left[\begin{array}{c}A_{1} X_{1} \\ 1 \\ 0 \\ 0\end{array}\right]-C_{1} X_{1}=-5 x_{1}-4 x_{1}-M$.
Thus, the corresponding linear programming is Min $w_{1}=-5 x_{1}-4 x_{1}-M$, Subject to 


$$
\hat{x}_{11}=(0,40), w_{1}^{*}=-160-M .
$$

For sub problem 2 where $j=2$

$$
Z_{2}-C_{2}=C_{B} B^{-1}=\left[\begin{array}{c}
A_{2} X_{2} \\
0 \\
1 \\
0
\end{array}\right]-C_{2} X_{2}=-M .
$$

Thus, the corresponding linear programming is

$\operatorname{Min} w_{2}=-M$,

Subject to

$$
\begin{aligned}
& 5 x_{3}+x_{4} \leq 12, \\
& x_{3}, x_{4} \geq 0, \\
& \hat{x}_{21}=(0,0), w_{2}^{*}=-M .
\end{aligned}
$$

For sub problem 3 where $j=3$

$$
Z_{3}-C_{3}=C_{B} B^{-1}=\left[\begin{array}{c}
A_{3} X_{3} \\
0 \\
0 \\
1
\end{array}\right]-C_{3} X_{3}=x_{5}-x_{6}-M .
$$

Thus, the corresponding linear programming is

$\operatorname{Min} w_{3}=-x_{5}-x_{6}-M$,

Subject to

$$
\begin{aligned}
& x_{5}+x_{6} \leq 20, \\
& x_{5}+5 x_{6} \leq 80, \\
& x_{5}, x_{6} \geq 0 . \\
& \hat{x}_{31}=(20,0), w_{3}^{*}=-20-M .
\end{aligned}
$$

Now, to determine new basic variable $w_{1}^{*}<w_{2}^{*} \& w_{1}^{*}<w_{3}^{*}$ $\& w_{1}^{*}<0$, so $\beta_{11}$ associated with $\hat{x}_{11}$ should enter basic solution and $x_{8}$ will leave basic solution.

After 4 iterations the FLDM obtain his optimal solution $\left(x_{1}^{F}, x_{2}^{F}, x_{3}^{F}, x_{4}^{F}, x_{5}^{F}, x_{6}^{F}\right)=(0,30,0,0,20,0)$.

Now set $\left(x_{1}, x_{2}\right)=(0,30)$ to the SLDM constraints.

Secondly, the SLDM solves his/her problem as follows:

$\operatorname{Max} F_{2}=\operatorname{Max} 8 x_{3}+4 x_{4}+x_{5}+x_{6}$,

Subject to

$$
\begin{aligned}
& x_{3}+x_{4}+x_{5}+x_{6} \leq 20, \\
& 5 x_{3}+x_{4} \leq 12, \\
& x_{5}+x_{6} \leq 20, \\
& x_{5}+5 x_{5} \leq 80, \\
& x_{3}, x_{4}, x_{5}, x_{6} \geq 0 . \\
& x^{2}
\end{aligned}
$$

The SLDM do the same action like FLDM till he obtains the optimal solution $\left(x_{3}^{S}, x_{4}^{S}, x_{5}^{S}, x_{6}^{S}\right)=(0,12,8,0)$, now set $\left(x_{3}, x_{4}\right)=(0,12)$ to the TLDM constraints.

Finally, the TLDM solves his/her problem as follows:

$\operatorname{Max}_{3}=\operatorname{Max} 8 x_{5}+6 x_{6}$,

Subject to

$$
\begin{aligned}
& x_{5}+x_{6} \leq 8, \\
& x_{5}+x_{6} \leq 20, \\
& x_{5}+5 x_{6} \leq 80, \\
& x_{5}, x_{6} \geq 0 .
\end{aligned}
$$

The TLDM do the same action like FLDM and SLDM till he obtain the optimal solution $\left(x_{5}^{T}, x_{6}^{T}\right)=(8,0)$.

So $\left(x_{1}^{F}, x_{2}^{F}, x_{3}^{S}, x_{4}^{S}, x_{5}^{T}, x_{6}^{T}\right)=(0,30,0,12,8,0)$ is the optimal solution for three level large scale linear programming problem, where $F_{1}=128, F_{2}=56$ and $F_{3}=64$.

\section{Conclusion}

In this paper, we presented a three level large scale linear programming problem in which the objective functions at every level are to be maximized. A three level programming problem can be thought as a static version of the Stackelberg strategy. An algorithm for solving a three-planner model and a solution method for treating this problem were suggested. At each level we attempted to optimize its problem separately as a large scale programming problem using Dantzig and Wolfe decomposition method. Therefore, we handle the optimization process through a series of sub problems that can be solved independently. Finally, a numerical example was given to clarify the main results developed in this paper. However, there are many other aspects, which should by explored and studied in the area of a large scale multi-level optimization such as:

1- Large scale multi-level non-linear programming problem with fuzzy parameters in the objective functions and in the constraints and with integrality conditions.

2- Large scale multi-level non-linear programming problem with stochastic parameters in the objective functions and in the constraints and with integrality conditions.

3- Large scale multi-level non-linear programming problem with rough parameters in the objective functions and in the constraints and with integrality conditions.

\section{References}

[1] T. Abou - El- Enin, on the solution of a special type of large scale integer linear vector optimization problems with uncertainty data through TOPSIS approach, Applied Mathematical Sciences, 3095-3105 (2010).

[2] T. Abou - El- Enin, on the solution of a special type of large scale linear fractional multiple objective programming problems with uncertainty data, International Journal of Contemporary Mathematical Sciences, 657-669 (2011).

[3] M.A. Abo-sinna, and Abou - El- Enin, An interactive algorithm for large scale multiple objective programming problems with fuzzy parameters through TOPSIS approach, Yugoslav Journal of Operations Research, 253-273 (2011).

[4] M.A. Abo-sinna, A. H. Amer and A. S. Ibrahim, Extensions of TOPSIS for large scale multi-objective non-linear programming problems with block angular structure, Applied Mathematical Modeling, 292-302 (2008).

[5] M. Balinski, An Algorithm For Finding All Vertices of Convex Polyhedral Sets, SIAM Journal, 9, 72-88 (1961).

[6] M. Benzi, E. Haber, and L. R. Hansson. Multilevel algorithms for large scale interior point methods in bound constraint optimization. Technical report, Emory University, Atlanta, (2006).

[7] G. Dantzig, and P. Wolfe, The decomposition algorithm for linear programs, Econometrics, 9, 767-778 (1961).

[8] S. Ding, Construction of multi-level electrical and electronic practice system, Journal of Theoretical and Applied Information Technology, 47, 679-686 (20th January 2013). 
[9] A. A. El-Sawy, N.A. El-khouly, and T.H.M. Abou - El- Enin, An algorithm for decomposing the parametric space in large scale linear vector optimization problems : A fuzzy approach, Advances in Modeling \& Analysis, 55, 1-16 (2000).

[10] O. Emam, Interactive approach to bi-level integer multi-objective fractional programming problem, Applied Mathematics and Computation, 223, 17-24 (2013).

[11] A. Gaur and S. R. Arora, Multi-level multi-Objective integer linear programming problem, Advanced Modeling and Optimization, 10, 297-322 (2008).

[12] M.S. Osman, O.M. Saad, and A.G. Hasan, Solving special class of large scale fuzzy multi objective integer linear programming problems, Fuzzy Sets and Systems, 107, 289297 (1999).

[13] S. Pramanik and D. Banerjee, Chance constrained quadratic bi-level programming problem, International Journal of Modern Engineering Research, 2, 2417-2424 (2012).

[14] S. Pramanik, D. Banerjee and B. Giri, Chance constrained linear plus linear fractional bi-level programming problem, International Journal of Computer Applications, 56, 34-39 (2012).

[15] A. Ruziyeva, S. Dempe, Yager ranking index in fuzzy bilevel optimization, Artificial Intelligence Research, 2, 55-68 (2013).

[16] M. Saraj and N. Safaei, Solving bi-level programming problems on using global criterion method with an interval approach, Applied Mathematical Sciences, 6, 1135-1141 (2012).

[17] M. Saraj and N. Safaei1, Fuzzy linear fractional bi-level multi-objective programming problems, International Journal of Applied Mathematical Research, 4, 643-658 (2012).

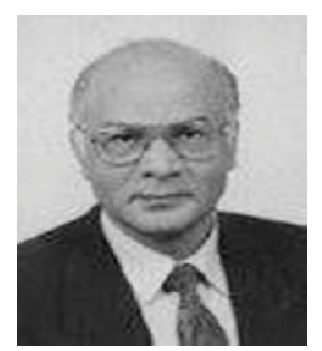

\section{Torkey Sultan \\ is a professor of information system} at Faculty of Computer Science and Information, Helwan University. He is referee of many journal in the field of information science. His research interest are in applied mathematics,

E-learning, and E-commerce.

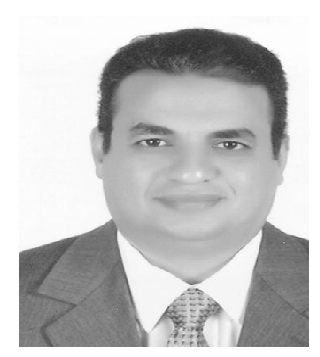

Osama Emam
is Associate professor
of information system
at Faculty of Computer
Science and Information,
Helwan University. He
received the Ph.D. degree in
operations research at Helwan
University. His research
interest in the multi-level

optimization field.

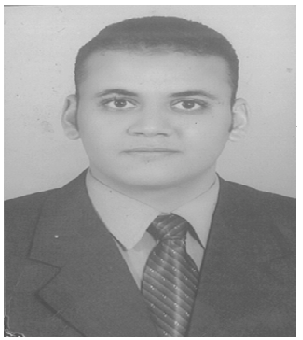

optimization field.

\author{
Amr Abohany \\ is a head department \\ of Electronic Document \\ Management Systems \\ at Telecom Egypt Company. \\ $\mathrm{He}$ received Bachelor \\ of Information Systems \\ at Zagazig University. \\ His research interest \\ in the multi-level large scale
}

